\title{
Traumatic cerebrospinal fluid leakage following septorhinoplasty
}

\author{
Ahmed Youssef ${ }^{1,2}$, Shahzad Ahmed ${ }^{1}$, Ahmed Aly Ibrahim² ${ }^{2}$ Mulvihill Daniel ${ }^{1}$, \\ Hisham M. Abdelfattah ${ }^{2}$, Haitham Morsi ${ }^{2}$ \\ ${ }^{1}$ Department of Otolaryngology-Head and Neck Surgery, University Hospital Birmingham, Birmingham, UK; ${ }^{2}$ Department of \\ Otolaryngology-Head and Neck Surgery, Alexandria University, Alexandria, Egypt
}

\begin{abstract}
Septoplasty/septorhinoplasty is a common ear, nose and throat procedure offered for those patients with deviated septum who are suffering from nasal obstruction and functional or cosmetic problems. Although it is a basic and simple procedure, it could lead to catastrophic complications including major skull base injuries which result in cerebrospinal fluid (CSF) leaks. We describe two different cases of traumatic CSF leaks following septoplasty/septorhinoplasty at two different sites. The first patient suffered a CSF leak following septoplasty and presented to Alexandria University Hospital. The leak was still active at presentation and identified as coming from a defect in the roof of the sphenoid sinus and was repaired surgically. The second patient presented 4 days after her cosmetic septorhinoplasty with a CSF leak and significant pneumocephalus. She was managed conservatively. Understanding the anatomical variations of the paranasal sinuses and implementing proper surgical techniques are crucial in preventing intracranial complications when performing either septoplasty or septorhinoplasty. A good quality computed tomography of the nose and paranasal sinuses is a valuable investigation to avoid major complications especially CSF leaks following either procedure.
\end{abstract}

Keywords Rhinoplasty / Cerebrospinal fluid rhinorrhea / Sphenoid sinus / Computed tomography / Diabetes insipidus
Correspondence: Ahmed Youssef Department of Otolaryngology-Head and Neck Surgery, University Hospital Birmingham, B152TH, UK

Tel: +201110003881

E-mail:

ahmedyouseef2002@yahoo.com
The article was presented in ALEXORL combined congress meeting April 2016(Alexandria, Egypt) and regional West Midland meeting October 2016 (Birmingham, UK). The Abstract is submitted for oral/poster presentation in AAO_HNSF on September 8-10, 2017, in Chicago, IL, USA.

\section{INTRODUCTION}

First documentation in 1757 from Quelmatz recommended daily digital pressure to the septum for gradual correction of a nasal deformity [1]. Many modifications have been suggested leading to the modern day septoplasty to be considered as one of the most common operations in otorhinolaryngology to treat the nasal obstruction. There are many complications post-septoplasty with complication rates of up to $18 \%$, including bleed- ing, infection, prolonged oedema, deformity and nasal airway obstruction. Rare complications include cavernous sinus thrombosis, meningitis, pneumocephalus, subarachnoid hemorrhage, periorbital emphysema, toxic shock syndrome, brain abscess and cerebrospinal fluid (CSF) leak [2].

CSF rhinorrhoea is a very rare complication and is normally due to damage of the cribriform plate after manipulation. Early recognition is important, so if spotted intraoperatively should be repaired at the time of surgery. If CSF has not been identified 
but suspected postoperatively, a computed tomography (CT) scan should be performed to rule out an intracranial complication with the identification of a pneumocephalus. Surgical management includes a way to close the leak and nonsurgical with bed rest, nasal packing with oral antibiotics. CSF leaks can sometimes resolve conservatively, however it is important to watch for meningitis related symptoms such as nuchal rigidity, headache, photophobia and fever $[2,3]$.

We are presenting two different cases of iatrogenic CSF leaks at two different sites. The first patient was presented to Alexandria University Hospital following septoplasty and he was repaired surgically. The second patient suffered a CSF leak following septorhinoplasty. She was admitted to another hospital and was managed conservatively.

\section{Fig. 1. Preoperative CT scan skull base bone window}

It shows a fracture at the level of the planum sphenoidale (red arrow) with multiple intracranial aeroceles (red star). CT, computed tomography.

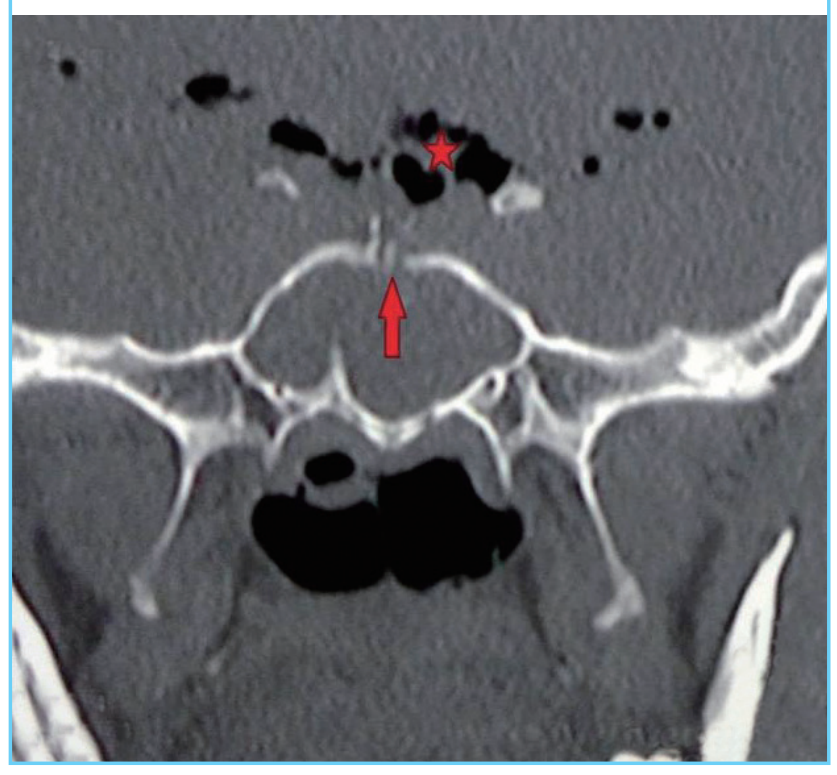

\section{CASES}

After taking an informed consent from presented patients for educational use and publication, we are presenting two different cases of iatrogenic CSF leaks at two different sites. The first patient was presented to our hospital following septoplasty and he was repaired surgically. The second patient suffered a CSF leak following septorhinoplasty. She was admitted to another hospital and was managed conservatively.

\section{Case 1}

It belongs to a 38-year-old male who underwent a septoplasty and was packed during the operation. Recovery was uneventful when the packs were removed on day 3 postoperatively. The patient started to develop CSF rhinorrhea, polydipsia and polyuria. The urine output was more than $2 \mathrm{~L}$ per hour and the plasma sodium level was $147 \mathrm{mmol} / \mathrm{L}$. A preliminary diagnosis of diabetes insipidus was considered. However, there was no evidence of meningitis. The endocrinology team recommended

\section{Fig. 2. Intraoperative image with a $0^{\circ}$ endoscope \\ It shows the breach in the skull base with the punctured dura com- ing into view (blue arrow).}

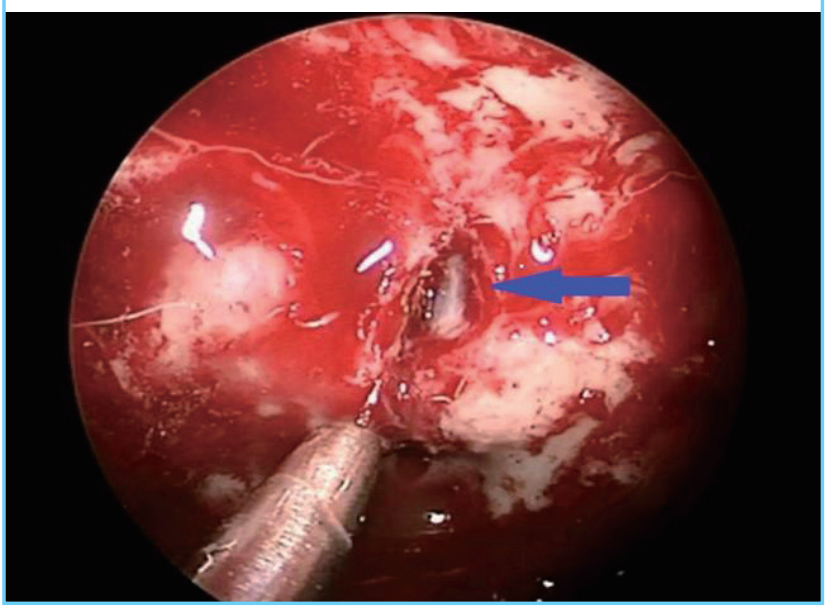

Fig. 3. Intraoperative endoscopic images during the surgical repair

It shows the multilayer repair using fat (A), fascia lata (B) and nasoseptal flap (C) respectively.
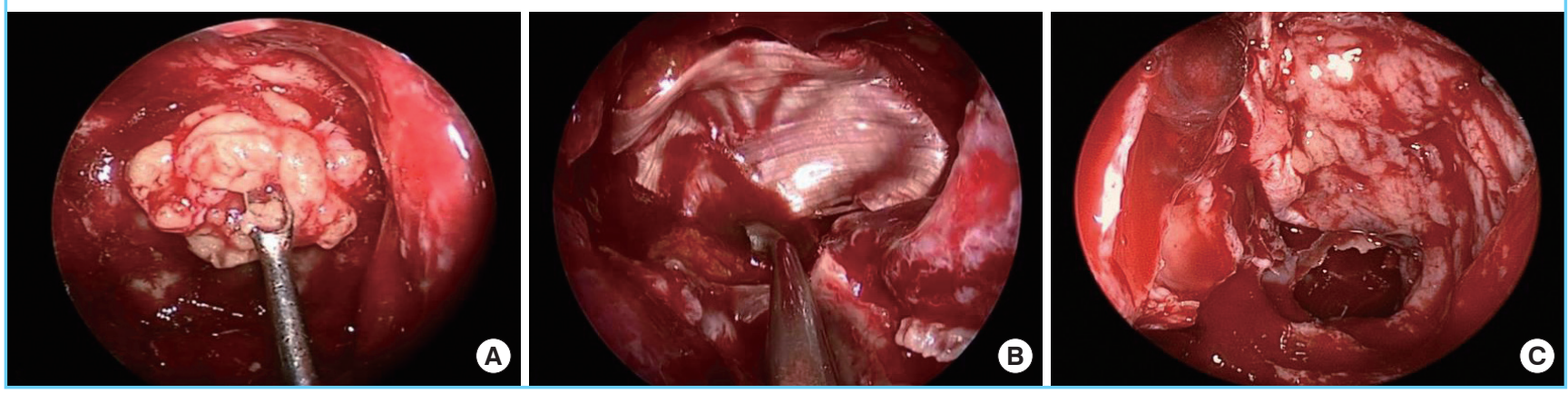


\section{Fig. 4. Postoperative sagittal T2-weighted MRI}

It shows the multilayered repair (red star) and enhanced nasoseptal flap over the repair (red arrow). MRI, magnetic resonance imaging.

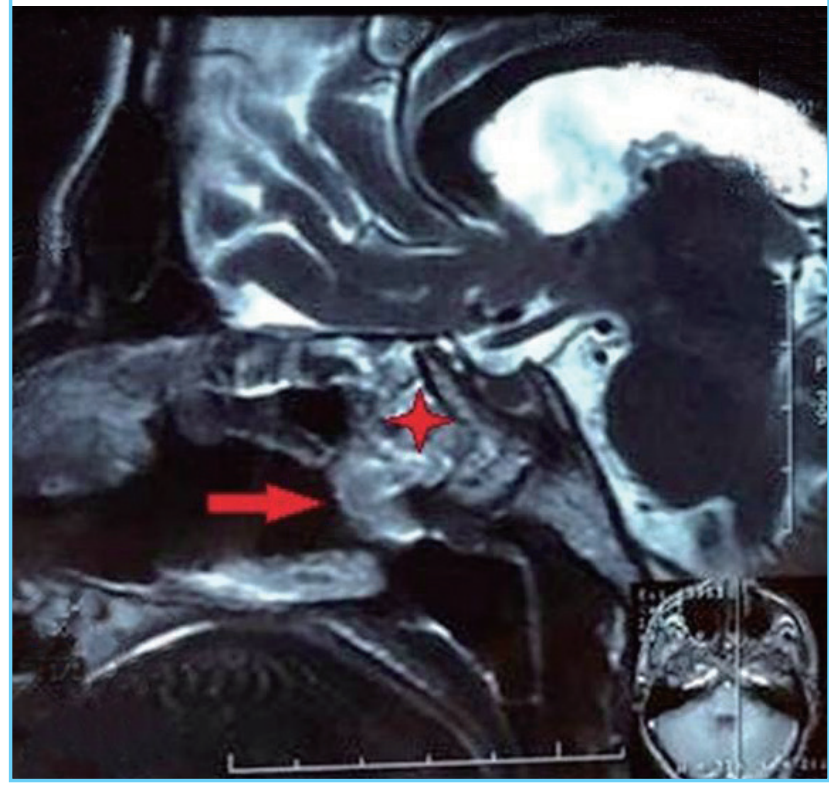

putting the patient on a therapeutic dose of desmopressin acetate for his diabetes insipidus. The patient was presented to us at our hospital for management of his condition. Further imaging study was recommended. A multislice CT of the nose, paranasal sinuses and skull base with intravenous contrast (IV) contrast was performed. It showed marked pneumocephalus in addition to a 7-mm defect in the roof of the sphenoid sinus that was continuous with the sellar CSF space (Fig. 1). There was no fracture of the cribriform plate. We underwent an endonasal endoscopic transsphenoidal approach to address the skull base defect that was found along the planum sphenoidale (Fig. 2). It was reconstructed in a multilayer fashion with fat, fascia lata in layers, nasoseptal flap, surgicel and tissue glue (Fig. 3). No further CSF leak was seen postoperatively. Magnetic resonance imaging (MRI) was ordered 3 months postoperatively and it showed nice successful repair of the skull base defect (Fig. 4).

A 6 months follow-up period of the patient showed no further CSF leakage from the nose and the urinary output was adequately controlled with medications.

\section{Case 2}

A 33-year-old female who underwent septorhinoplasty at a prestigious plastic surgery center was presented to another university hospital with a CSF leak. The leak started 5 days following her surgery. She complained of watery nasal discharge after removal of her nasal packs and this was associated with frequent attacks of severe headache. CT of the nose and paranasal sinuses

\section{Fig. 5. Axial CT scan for brain and sinuses}

It is done after removal of the nasal packs showing marked pneumocephalus (yellow arrows). CT, computed tomography.

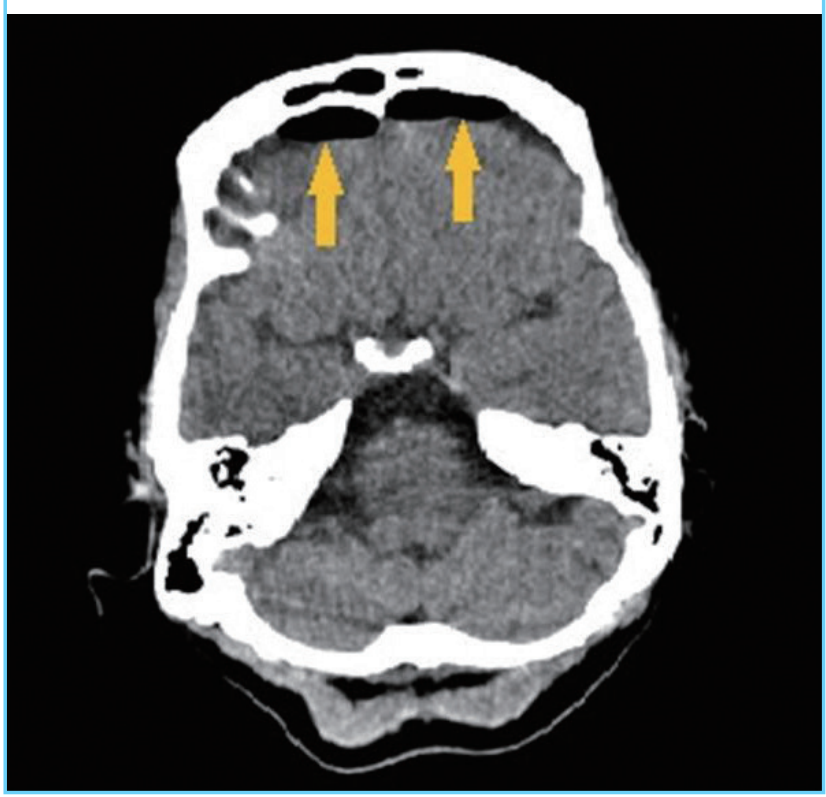

was done at another hospital which showed marked pneumocephalus (Fig. 4). The patient was admitted and further imaging studies were requested. The CT/MRI images of the paranasal sinuses and skull base did not show an obvious site of skull base fracture or breach (Figs. 5 and 6). Review of her operative details through communication with her operating surgeon did not reveal unusual intraoperative events or immediate postoperative mishaps. Careful observation and conservative measures were offered to the patient that included bed rest, lying flat and IV prophylactic antibiotics. Fortunately, no further leakage was detected and the pneumocephalus was resolved. The patient was sent home few days later without the need of any surgical intervention. A follow-up period of 6 months was recommended during which the condition was stable and no CSF leakage was detected in that period.

\section{DISCUSSION}

Post-septoplasty CSF leak is very rare. Its known cause is due to faulty surgical techniques in handling the perpendicular plate of ethmoid bone [3]. There are only four cases of CSF rhinorrhoea has been mentioned in the literature which occurred postseptoplasty [4]. None however, show involvement of the roof of the sphenoid sinus as in the case study reported.

As frequently reported, the most likely point of injury is between the ethmoid labyrinth and cribriform plate where the dura is tightly adherent called the lateral lamella [5]. However, 


\section{Fig. 6. MRI T2-weighted images}

(A) Coronal view and (B) sagittal view. It shows marked pneumocephalus with no obvious meningoencephalocele along the skull base (yellow arrow). MRI, magnetic resonance imaging.

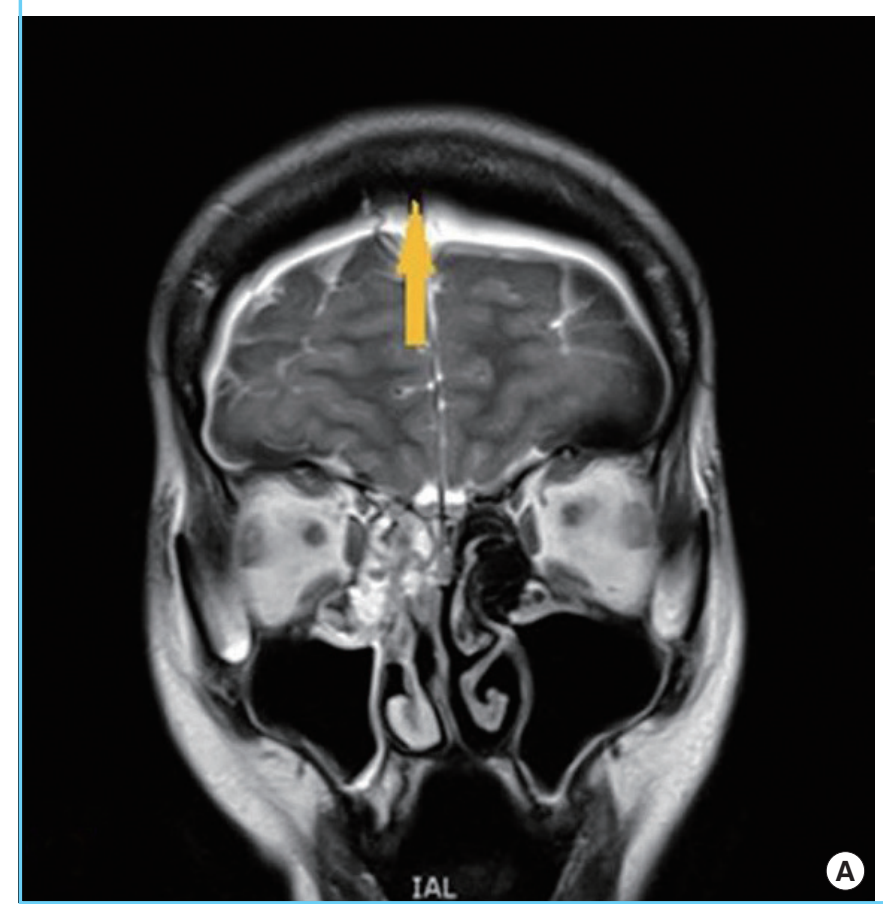

the posterior parts of the perpendicular plate of vomer and ethmoid anatomically are linked with the anterior aspect of the sphenoid sinus. If there is incorrect manipulation to this region it can cause a fracture in the sphenoid sinus roof or inter-sphenoid septum [6].

The literature emphasizes against the twisting motion of the perpendicular plate of ethmoid which was identified by Onerci, et al. [7] in 2004, but it rarely explains that this could lead to severe complications involving the cribriform plate.

It is important to evaluate the levels of the ethmoid roof due to patient variations. The distance of the highest level of the ethmoid labyrinth and cribriform plate at anterior cranial fossa can vary from 0.6 to $11.7 \mathrm{~mm}$ and from the floor of the nasal cavity to the cribriform plate 38 to $52 \mathrm{~mm}$.

Forces applied to the perpendicular plate of ethmoid would transmit to the weak and thin cribriform plate and elicit a micro fracture. Instead of twisting and fracturing the perpendicular plate the use of turbinate or septum scissors to remove this bone will prevent multidirectional force on the cribriform plate [8].

In the reported case, the skull base defect was at the roof of the sphenoid sinus along the planum sphenoidale. The fractured bone spicules penetrated the dura and the subarachnoid space to involve the pituitary stalk which was detected intraoperatively with $0^{\circ}$ nasal endoscope. This clearly explains how diabetes insipidus occurred.

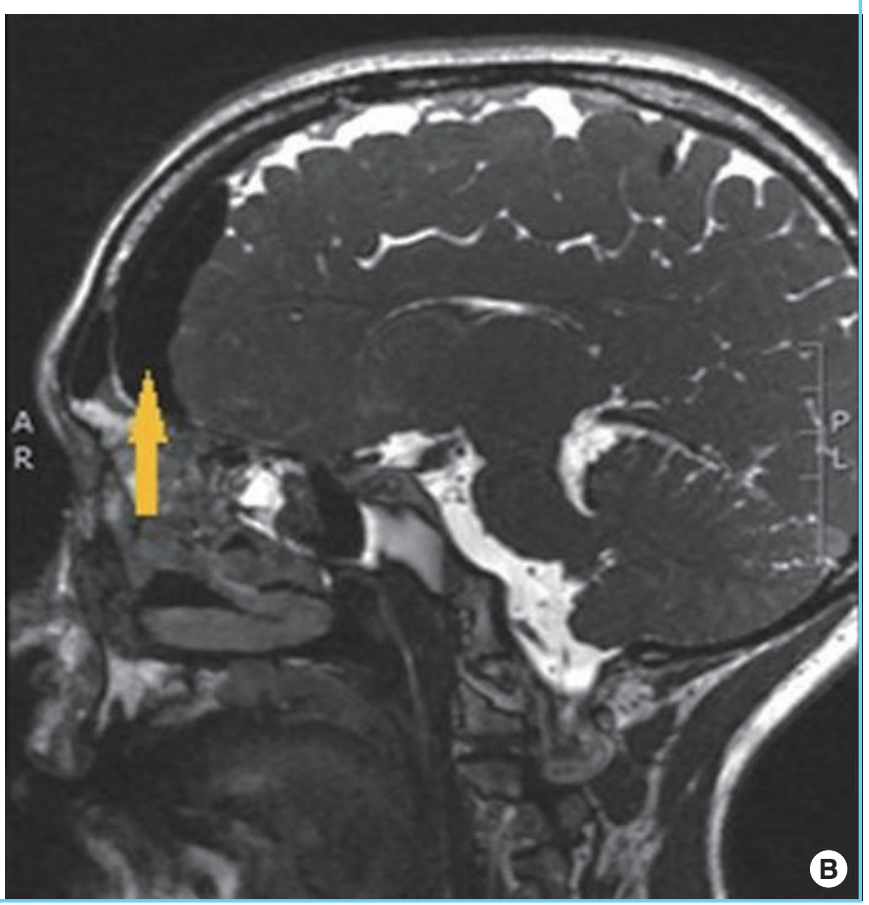

It is important to note that even though there is no visible fracture at the cribriform plate on a CT scan, a pneumocephalus is indicative that there has been a breach in the skull base. This could be as a result of sneezing or coughing when the intranasal pressure is high enough to breach the cribriform plate without showing a fracture on the scan [3].

Regarding our reported case, a multislice CT of the nose, paranasal sinuses and skull base showed pneumocephalus with a breach in skull base along the roof of the sphenoid sinus with evident fracture in the inter-sphenoid septum which was continuous with the breached area. No violation was detected along the cribriform plate. In regards of the operative notes of this case, the surgeon used a chisel and a hammer to remove a septal spur over the floor near to bony-cartilaginous junction. The surgery went smoothly without intraoperative leakage or massive bleeding. A CT of nose and paranasal sinuses was not performed before the surgery.

Intraoperative findings confirmed the presence of a skull base defect over the planum sphenoidale with gushes of CSF coming from the subarachnoid space through a dural tear. Navigation using $0^{\circ}$ scope was performed and showed a part of pituitary stalk and the floor of the third ventricle. This explained the possibility of injury to the pituitary stalk which resulted in diabetes insipidus.

Multilayer repair of the CSF leakage was done using fat har- 
vested from the thigh to plug the dural defect. After adequate removal of the mucosal lining of sphenoid sinus, we used two layers of fascia lata to reconstruct the skull base defect followed by a nasoseptal flap which was raised and laid down over the repair to support it. We packed the sphenoid sinus with surgicel that was held in place with tissue glue.

Finally, the nose was packed with two nasopores. Follow-up of the case was done and no further leak was detected. No symptoms or signs of meningitis were experienced. MRI with gadolinium was done 1 month following the surgery, which showed adequate repair with enhancing septal flap. However, the patient symptoms and signs of diabetes insipidus have not shown any improvement. He is completely dependent on the same dose of desmopressin, which might suggest an irreversible damage to his pituitary stalk and injury to his neuro-hypophysis tract.

However, conservative management was a good option in the second post-rhinoplasty case, where no obvious site of leak was detected. Strict bed rest and IV antibiotics were very essential to prevent possible meningitis. We followed the same strategy as in cases of post-traumatic CSF leakage following skull base fractures [9] and it did help to prevent further leakage. "Watch and wait" policy should always be considered when there is no detectable skull base defect.

In both cases, early detection of CSF leak following either surgery helped a lot. We followed the right protocol and avoided further co-morbidities like meningitis or fatal electrolyte imbalance resulting from diabetes insipidus.

\section{NOTES}

\section{Conflict of interest}

No potential conflict of interest relevant to this article was reported.

\section{Ethical approval}

The study was performed in accordance with the principles of the Declaration of Helsinki. Written informed consents were obtained.

\section{Patient consent}

The patients provided written informed consent for the publication and the use of their images.

\section{REFERENCES}

1. Hinderer KH. History of septoplasty. In: Hinderer KH, editor. Fundamentals of anatomy and surgery of the nose. Birmingham: Aesculapius Publ. Co.; 1971.

2. Wax MK, Ramadan HH, Ortiz O, et al. Contemporary management of cerebrospinal fluid rhinorrhea. Otolaryngol Head Neck Surg 1997;116:442-9.

3. Soni RS, Choudhry OJ, Liu JK, et al. Postoperative cerebrospinal fluid leak after septoplasty: a potential complication of occult anterior skull base encephalocele. Allergy Rhinol (Providence) 2013;4:e41-4.

4. Venkatesan NN, Mattox DE, Del Gaudio JM. Cerebrospinal fluid leaks following septoplasty. Ear Nose Throat J 2014; 93:E43-6.

5. Gray ST, Wu AW. Pathophysiology of iatrogenic and traumatic skull base injury. Adv Otorhinolaryngol 2013;74:1223.

6. Guvenc G, Eren E, Arslanoglu S, et al. A rare complication of septoplasty: tension pneumocephalus without rhinorrhea. J Craniofac Surg 2014;25:e360-1.

7. Onerci TM, Ayhan K, Ogretmenoglu O. Two consecutive cases of cerebrospinal fluid rhinorrhea after septoplasty operation. Am J Otolaryngol 2004;25:354-6.

8. Al Arfaj A. A rare complication of septorhinoplasty. Plast Reconstr Surg Glob Open 2014;2:e273.

9. Friedman JA, Ebersold MJ, Quast LM. Post-traumatic cerebrospinal fluid leakage. World J Surg 2001;25:1062-6. 